\title{
Passive Front Propagation in Intense Turbulence: Early Transient and Late Statistically Stationary Stages of the Front Area Evolution
}

\author{
Vladimir A. Sabelnikov ${ }^{1,2}$ and Andrei N. Lipatnikov $3, *$ (i) \\ 1 DMPE ONERA, University of Paris-Saclay, F-91123 Palaiseau, France; sabelnikov@free.fr \\ 2 Central Aerohydrodynamic Institute (TsAGI), 140180 Zhukovsky, Russia \\ 3 Department of Mechanics and Maritime Sciences, Chalmers University of Technology, \\ 41296 Gothenburg, Sweden \\ * Correspondence: lipatn@chalmers.se
}

check for

updates

Citation: Sabelnikov, V.A.; Lipatnikov, A.N. Passive Front Propagation in Intense Turbulence: Early Transient and Late Statistically Stationary Stages of the Front Area Evolution. Energies 2021, 14, 5102. https://doi.org/10.3390/en14165102

\section{Academic Editors:}

Nilanjan Chakraborty and

Bjørn H. Hjertager

Received: 18 July 2021

Accepted: 11 August 2021

Published: 19 August 2021

Publisher's Note: MDPI stays neutral with regard to jurisdictional claims in published maps and institutional affiliations.

Copyright: (c) 2021 by the authors. Licensee MDPI, Basel, Switzerland. This article is an open access article distributed under the terms and conditions of the Creative Commons Attribution (CC BY) license (https:/ / creativecommons.org/licenses/by/ $4.0 /)$.

\begin{abstract}
The influence of statistically stationary, homogeneous isotropic turbulence (i) on the mean area of a passive front propagating in a constant-density fluid and, hence, (ii) on the mean fluid consumption velocity $\bar{u}_{T}$ is explored, particularly in the case of an asymptotically high turbulent Reynolds number, and an asymptotically high ratio of the Kolmogorov velocity to a constant speed $u_{0}$ of the front. First, a short early transient stage is analyzed by assuming that the front remains close to a material surface that coincides with the front at the initial instant. Therefore, similarly to a material surface, the front area grows exponentially with time. This stage, whose duration is much less than an integral time scale of the turbulent flow, is argued to come to an end once the volume of fluid consumed by the front is equal to the volume embraced due to the turbulent dispersion of the front. The mean fluid consumption velocity averaged over this stage is shown to be proportional to the rms turbulent velocity $u^{\prime}$. Second, a late statistically stationary regime of the front evolution is studied. A new length scale characterizing the smallest wrinkles of the front surface is introduced. Since this length scale is smaller than the Kolmogorov length scale $\eta_{K}$ under conditions of the present study, the front is hypothesized to be a bifractal with two different fractal dimensions for wrinkles larger and smaller than $\eta_{K}$. Finally, a simple scaling of $\bar{u}_{T} \propto u^{\prime}$ is obtained for this late stage as well.
\end{abstract}

Keywords: self-propagating front; turbulent consumption velocity; front area; bifractal

\section{Introduction}

Turbulent combustion involves various multi-scale and highly non-linear phenomena [1-3] such as turbulence [4-6], complex chemistry [7,8], thermal expansion [9-12], and differential diffusion [13] effects. Nevertheless, the fundamentals of the influence of turbulence on a flame are often explored by considering the classical problem of a passive front propagating locally normal to itself at a constant speed $u_{0}$ in randomly turbulent advected media [14-16]. Under such simplified conditions, the front speed is associated with the laminar flame speed. Historically, this problem attracted much attention, particularly in the 1940s when significant acceleration of flame propagation by turbulence was found. The phenomenon was explained by Damköhler [17] and Shelkin [18] who highlighted random advection of a flame by turbulent flow and reduced the influence of the turbulence on the flame to an increase in the area of the flame surface wrinkled due to large-scale velocity fluctuations. Following these pioneering ideas, various models of flame propagation in turbulent flow were created in order to evaluate the mean consumption velocity $\bar{u}_{T}$ (i.e., the averaged mass rate of reactant consumption per unit area of the mean flame surface, normalized using the fluid density upstream of the flame). The mean consumption velocity was considered to be a function of the front speed $u_{0}$ and the rms turbulent velocity $u^{\prime}$, with a ratio of $\bar{u}_{T} / u_{0}$ being controlled by the mean increase in the front surface area. Moreover, 
Direct Numerical Simulation (DNS) investigation [19] of the propagation of a dynamically passive front in a constant-density turbulent flow showed the linear relationship between $\bar{u}_{T}-u_{0}$ and $u^{\prime}$, at least at $0.5 \leq u^{\prime} / u_{0} \leq 10$.

However, in spite of long-term investigations of propagation of a front (e.g., a flame) in randomly advected media (e.g., turbulence), physical mechanisms that result in the aforementioned linear relation do not seem to be fully clarified. To resolve the problem, turbulent entrainment-which is controlled by large-scale eddies-is commonly highlighted, with small-scale characteristics of any surface (material or self-propagating) being assumed to be adjusted to the influence of large-scale turbulent eddies on the surface. Accordingly, the fractal concept [20] is invoked to describe the surface characteristics at various scales. In particular, for a moderately slow $\left(u_{K}<u_{0} \ll u^{\prime}\right)$ front whose fractal dimension $D=7 / 3$ and the Gibson length scale $L_{G}=L\left(u_{0} / u^{\prime}\right)^{3} \ll L$ is inside the inertial interval of the turbulence spectrum [4,5], i.e., $\eta_{K}<L_{G}$, the fractal concept yields $\bar{u}_{T} \propto u^{\prime}$ [21,22]. Here, $L$ is an integral turbulence length scale, $u_{K}=(v \varepsilon)^{1 / 4}$ and $\eta_{K}=\left(v^{3} / \varepsilon\right)^{1 / 4}$ designate Kolmogorov velocity and length scales [4], respectively, $v$ is the kinematic viscosity of the fluid, and $\varepsilon$ is the mean rate of viscous dissipation of turbulent kinetic energy. However, it is an open question what happens when $u_{0} / u_{K} \rightarrow 0$ and, consequently, the Gibson length scale is inside the viscous (dissipation) interval, i.e., $\eta_{K}>L_{G}$. Accordingly, the present work aims to hypothesize a specific physical mechanism that reconciles (i) the scaling of $\bar{u}_{T} \propto u^{\prime}$ at $u_{0} \ll u_{K}$, (ii) the concept of turbulent entrainment, and (iii) a well-recognized paradigm that reduces the effect of turbulence on the front propagation to an increase in the front area by turbulent eddies, whose characteristics are described by the Kolmogorov theory $[4,5,23]$.

The present study addresses two limiting stages of front area evolution: (i) an early transient stage, whose duration is much less than an eddy-turn-over time scale $\tau_{T}=L / u^{\prime}$, and (ii) a late stage when the front area reaches a statistically stationary state. The two stages could be associated with (i) flame kernel growth after spark ignition in a piston engine, and (ii) a fully developed turbulent premixed flame, respectively. During the late stage, growth of the front surface area due to turbulent straining is counterbalanced by a reduction of the front surface area due to joint actions of (i) the folding of finite-length front elements, caused by strong advection, and (ii) the subsequent collisions of self-propagating fronts.

\section{Analysis and Results}

\subsection{Earlier Transit Stage}

Let us explore the motion of an infinitely thin front in statistically stationary, homogeneous, isotropic turbulence. The front self-propagates locally normal to itself at a constant speed $u_{0}$. The turbulence is (i) unaffected by the front, (ii) characterized by an asymptotically high turbulent Reynolds number $R e_{L}=u^{\prime} L / v \gg 1$, and (iii) described by the Kolmogorov theory $[4,5,23]$. The front is considered to propagate slowly, i.e., the Kolmogorov velocity $u_{K}$ is much larger than $u_{0}$. In this section, we address the early transient stage, i.e., $t \ll \tau_{T}$, of the evolution of an initially ( $\left.t=0\right)$ planar front.

The present analysis is based on the theory of surface area growth, developed by Batchelor [24] for an infinitesimal element of a material surface. The analysis is also based on the results of DNS studies [25,26] of the same phenomenon, and theoretical and DNS results [27] on the growth of the area of a finite-length element of a material surface, i.e., an element whose area is much larger than $L^{2}$. We also use the theory of turbulent diffusion, developed by Taylor [28]. As we will see later, the earlier transit stage takes a time interval much shorter than the eddy turnover time $\tau_{T}$. During such a short time interval, the area growth rates are almost the same for the infinitesimal and finite-length elements of a material surface [27]. This is because the folding of the finite-length elements, caused by large-scale eddies, is a relatively slow process.

When a planar material surface-which is normal to the $x$-axis (streamwise direction in the following) - is embedded into the Kolmogorov turbulence, the surface adapts itself to the flow field during a short transient time interval of $t \geq t_{i} \approx(2.5-3) \tau_{K}$. Subsequently, 
the mean (ensemble-averaged) surface area $A_{M}(t)$ is expected to grow exponentially with time [24-26], i.e.,

$$
A_{M}(t)=A_{0} \exp \left(\frac{\xi t}{\tau_{K}}\right),
$$

where $\xi$ is a constant at about $0.28[25,26], \tau_{K}=(\nu / \varepsilon)^{1 / 2}$ designates the Kolmogorov time scale, and $A_{0} \gg L^{2}$ is the area of the considered element of the initial planar material surface at $t=0$, i.e., $A_{0}=A_{M}(0)$. Note that a subsequent DNS study [27] indicates that, due to the folding of finite-length material surface elements during a later stage, $\xi=0.345+0.00525 R e_{L}^{1 / 2}$ in a range of $10<R e_{L}^{1 / 2}<25$ when $t \approx \tau_{T}$.

At the same time, the streamwise dispersion $\Delta_{M}(t)$ of a material surface grows linearly with time [28].

$$
\Delta_{M}(t)=u^{\prime} t
$$

at $0<t \ll \tau_{T}$, with a similar linear dependence of a mean turbulent flame brush thickness on flame-development time being documented in various experiments reviewed elsewhere [29]. The constraints of $t \geq t_{i} \approx(2.5-3) \tau_{K}$ and $t \ll \tau_{T}$ are consistent with one another in the considered case of $R e_{L} \gg 1$.

As argued by Yeung et al. [26], Equation (1), which holds for an infinitesimal element of a material surface, describes also the evolution of the area $A_{F}(t)$ of an infinitesimal element of a dynamically passive front, provided that $u_{0} \ll u_{K}$ and $t \geq t_{i} \approx(2.5-3) \tau_{K}$ Moreover, during the studied short earlier stage $\left(t \ll \tau_{T}\right)$, the same equation holds for finite-length surface elements [27], as already noted earlier. Thus, if $u_{0} \ll u_{K}$

$$
\begin{gathered}
A_{F}(t)=A_{0} \exp \left(\frac{\xi t}{\tau_{K}}\right), \\
\Delta_{F}(t)=u^{\prime} t
\end{gathered}
$$

at $t_{i}<t \ll \tau_{T}$.

Furthermore, the already cited DNS study by Yeung et al. [26] shows that, during the considered time interval $\left(0<t \ll \tau_{T}\right)$, the distance between the material and the self-propagating surfaces that coincide at $t=0$ is smaller than the Kolmogorov length scale $\eta_{K}$ with a high probability. This feature is associated with the well-known fact that positive rates of strain of a material surface statistically dominate in the Kolmogorov turbulence. Indeed, if the local rate of strain of a material surface is positive, the locally normal (to the surface) velocity vector $\mathbf{u}_{n}$ points to the surface, and the magnitude $\left|\mathbf{u}_{n}\right|$ is increased with distance from the surface. Consequently, the velocity $\left|\mathbf{u}_{n}\right|$ can be significantly larger than $u_{0} \ll u_{K}$ already at a short distance from the surface. Therefore, as $u_{0} \ll\left|\mathbf{u}_{n}\right|$, selfpropagation of the front plays a minor role locally, the vector $\mathbf{u}_{n}+u_{0} \mathbf{n} \approx \mathbf{u}_{n}$ points to the front, and the local flow impedes further divergence of the front and material surface.

However, there are fundamental differences between the two surfaces. Indeed, first, there is no cusp formation at a material surface and, second, the neighboring/adjoining elements of a folded (folds are produced by strong advection) material surface never collide. Therefore, (i) the area of a material surface is well known to grow exponentially with time, and (ii) the distance $d$ between the surface elements can be very low. For instance, DNS data show that the distance $d$ is randomly distributed in a wide range of length scales, which can be seen in Figure 6 in Ref. [26]. In the case of a self-propagating front, cusp formation and collisions of its elements result in the local surface annihilation if the local distance between the neighboring front elements is small enough. However, during the studied short earlier stage, both effects may be neglected, as discussed earlier. 
Let us compare the fluid volume consumed by the front at instant $t$ with the volume of the streamwise turbulent dispersion of the front, i.e., a volume bound by the leading and trailing edges of the front. The former volume can be estimated as follows:

$$
V_{F}(t)=u_{0} \int_{t_{i}}^{t} A_{F}(\theta) d \theta=u_{0} A_{0} \int_{t_{i}}^{t} \exp \left(\xi \frac{\theta}{\tau_{K}}\right) d \theta=u_{0} \tau_{K} \xi^{-1} A_{0}\left[\exp \left(\xi \frac{t}{\tau_{K}}\right)-\exp \left(\xi \frac{t_{i}}{\tau_{K}}\right)\right],
$$

where $u_{0} A_{F}(\theta)$ is the volume rate of the fluid consumption at instant $\theta$.

If $t_{i} \ll t$, the second term in square brackets is negligible, and

$$
V_{F}(t) \approx u_{0} \tau_{K} \xi^{-1} A_{0} \exp \left(\frac{\xi t}{\tau_{K}}\right)
$$

i.e., the volume of the consumed fluid is controlled by the small-scale turbulence and grows exponentially with time.

By virtue of Equation (4), the volume of the streamwise dispersion of the front is equal to

$$
V_{T}(t)=A_{0} \Delta_{F}(t) \propto A_{0} u^{\prime} t
$$

and, consequently, is controlled by large-scale turbulent eddies. This volume grows linearly with time at $0<t \ll \tau_{T}$, contrary to the exponential growth of $V_{F}(t)$. Therefore, in spite of $V_{F}(t) \ll V_{T}(t)$ at $\xi t / \tau_{K}=\mathrm{O}(1)$, because $u_{0} \ll u_{K} \ll u^{\prime}$, the exponentially growing volume $V_{F}(t)$ and the linearly growing volume $V_{T}(t)$ should become equal to one another at certain instant $t^{*}$. In other words, at instant $t^{*}$, the fluid consumed by the front fills the volume formed by the streamwise dispersion of the front.

To estimate this critical instant, let us invoke the following simple constraint:

$$
V_{F}\left(t^{*}\right)=V_{T}\left(t^{*}\right)
$$

Henceforth, numerical factors are skipped for simplicity. Equation (8) can be rewritten as follows:

$$
\frac{V_{F}\left(t^{*}\right)}{A_{F}\left(t^{*}\right)}=l\left(t^{*}\right)=d\left(t^{*}\right)=\frac{V_{T}\left(t^{*}\right)}{A_{F}\left(t^{*}\right)}
$$

Here, $l\left(t^{*}\right)$ is the mean thickness of a thin layer consumed by the front, or, in other words, the mean distance between initially coinciding elements of the front and material surface. The symbol $d\left(t^{*}\right)$ designates the mean distance between opposed elements of either the front or the material.

Equations (3) and (6) yield

$$
\begin{gathered}
l\left(t^{*}\right)=\xi^{-1} \ell_{0} \\
\ell_{0}=u_{0} \tau_{K}=\frac{u_{0}}{u_{K}} \eta_{K} \ll \eta_{K} .
\end{gathered}
$$

Both the distance $l\left(t^{*}\right)$ and the microscale $\ell_{0}$ are much less than the Kolmogorov length scale, i.e., they are inside the dissipation subrange of the turbulence spectrum. This estimate agrees with the DNS data by Yeung et al. [26], thus supporting the present study. Note that the microscale $\ell_{0}$ will also play an important role in an analysis of the statistically stationary state of the front evolution, discussed in the next subsection.

Substitution of Equations (6) and (7) into Equation (8), or substitution of Equations (1), (3), (6), and (7) into Equation (9) yields.

$$
\left(\frac{u^{\prime}}{u_{0}}\right)\left(\frac{\xi t^{*}}{\tau_{K}}\right) \approx \exp \left(\xi \frac{t^{*}}{\tau_{K}}\right)
$$

Taking logarithm of Equation (11), we arrive at

$$
\xi \frac{t^{*}}{\tau_{K}} \approx \ln \left(\frac{u^{\prime}}{u_{0}}\right)+\ln \left(\frac{\xi t^{*}}{\tau_{K}}\right)
$$


Under the considered conditions of $u_{0} \ll u_{K} \ll u^{\prime}$, term $\ln \left(u^{\prime} / u_{0}\right) \gg 1$. Therefore, $\xi t^{*} / \tau_{K} \gg 1$, the last term on the right-hand side of Equation (12) may be neglected when compared to the left-hand side. Consequently, the non-linear Equation (11) has the following approximate solution:

$$
t^{*} \approx \xi^{-1} \tau_{K} \ln \left(\frac{u^{\prime}}{u_{0}}\right) \approx \xi^{-1} \tau_{T} R e_{L}^{-1 / 2} \ln \left(\frac{u^{\prime}}{u_{0}}\right) .
$$

By virtue of Equation (13), the following necessary condition

$$
\ln \left(\frac{u^{\prime}}{u_{0}}\right) \ll R e_{L}^{1 / 2}
$$

should be satisfied in order for $t \ll \tau_{T}$, which is required for Equations (2) and (4) to be valid.

At instant $t^{*}$, the front area given by Equations (3) and (11) is equal to

$$
\frac{A_{F}\left(t^{*}\right)}{A_{0}}=\xi\left(\frac{u^{\prime}}{u_{0}}\right)\left(\frac{t^{*}}{\tau_{K}}\right) \approx\left(\frac{u^{\prime}}{u_{0}}\right) \ln \left(\frac{u^{\prime}}{u_{0}}\right) .
$$

The turbulent consumption velocity is equal to

$$
u_{T}\left(t^{*}\right)=u_{0} \frac{A_{F}\left(t^{*}\right)}{A_{0}}=\xi\left(\frac{t^{*}}{\tau_{K}}\right) u^{\prime} \approx u^{\prime} \ln \left(\frac{u^{\prime}}{u_{0}}\right) .
$$

The volume of the consumed fluid is equal to

$$
V_{F}\left(t^{*}\right)=A_{0} u^{\prime} t^{*}
$$

see Equations (7) and (8). Finally, the mean consumption velocity averaged over $0<t<t^{*}$ is equal to

$$
\bar{u}_{T}=\frac{V_{F}\left(t^{*}\right)}{A_{0} t^{*}}=u^{\prime} .
$$

Independence of the mean consumption velocity on the Kolmogorov scales does not mean that the Kolmogorov eddies are unimportant. On the contrary, it is the Kolmogorov eddies that create front surface within the framework of the above analysis. Nevertheless, the outcome, i.e., the mean $\bar{u}_{T}$, is independent of the Kolmogorov scales. This apparent paradox is basically similar to the well-known independence of the mean dissipation rate on viscosity in the Kolmogorov turbulence at $R e_{L} \rightarrow \infty$, or independence of the mean rate of entrainment of ambient irrotational fluid into turbulent fluid on viscosity in shear flows [30]. While both the dissipation and entrainment occur due to viscosity, the mean rates of the two processes are controlled by large-scale velocity fluctuations at $R e_{L} \rightarrow \infty$, whereas small-scale phenomena adjust themselves to these mean rates. As noted by Tsinober [6], "small scales do the 'work', but the amount of work is fixed by the large scales in such a way that the outcome is independent of viscosity".

\subsection{Statistically Stationary State}

The method used in Section 2.1 to analyze the early $\left(t \ll \tau_{T}\right)$ transient stage of front propagation under conditions of $u_{0} \ll u_{K} \ll u^{\prime}$ is based on the hypothesis that a material surface and a self-propagating front that coincide at $t=0$ are very close to one another (i.e., the distance between them is smaller than the Kolmogorov length scale) during a short $\left(t \ll \tau_{T}\right)$ time interval. This hypothesis allows us to model temporal growth of the front surface area by invoking results that are well known for material surfaces. However, this hypothesis does not hold at $t \gg \tau_{T}$ when the front area reaches a statistically stationary state. In this limit, the growth of the front surface area due to turbulent straining is counterbalanced by a reduction of the front surface area due to joint actions of folding 
of finite-length front elements, caused by strong advection, and subsequent collisions of self-propagating fronts. As a result, neighboring front surface elements collide, and the front surface area is reduced.

Here, to examine the statistically stationary regime of slow front propagation, we will show that the smoothing of small-scale wrinkles occurs in the dissipation range of the turbulence spectrum (i.e., at length scales smaller than the Kolmogorov scale). Accordingly, we will consider the front surface to be a bifractal, i.e., two fractals with different dimensions, associated with the dissipation and inertial ranges. A similar scenario was explored by Sreenevasan et al. [20] when discussing turbulent mixing for Schmidt numbers far greater than unity, as portrayed in Figures 2a and 6 in the cited paper. Recently, such ideas were developed for a flame of a finite thickness [31,32]. In the present communication, the bifractal concept is applied to an infinitely thin front. In particular, to explore the influence of turbulent eddies on the area of a slowly $\left(u_{0} \ll u_{K}\right)$ propagating front, the area response to small-scale and large-scale turbulent eddies is modeled by invoking two different fractal submodels. More specifically, both large-scale and small-scale wrinkles of the front are considered to be fractals, but with different dimensions $\left(D_{f, 1}\right.$ and $\left.D_{f, 2}\right)$ and different cut-off scales. Moreover, the outer cut-off scale for the small-scale fractal is considered to be equal to the inner cut-off scale for the large-scale fractal. These two equal cut-off scales are called a crossover length scale in the following. Thus, the focus of the following discussion is placed on the two fractal dimensions, the crossover length scale, as well as the inner $\ell_{\text {in }}$ and outer $\ell_{\text {out }}$ cut-off scales for small-scale and large-scale wrinkles of the front surface, respectively.

First, following a common supposition [20-22], the large outer cut-off scale $\ell_{\text {out }}$ is assumed to be proportional to a turbulent integral length scale $L$.

Second, the crossover length scale is associated with the boundary between inertial and dissipation ranges of the turbulence spectrum. Therefore, the crossover length scale is proportional to the Kolmogorov length scale $\eta_{K}$. Thus, the large-scale fractal covers the following range $\eta_{K}<r<L$ of wrinkle scales $r$. It is worth noting that $\eta_{K}$ is considered to be the inner cut-off scale not only in single-fractal models of non-reacting turbulent flows [20] or a bifractal model of turbulent mixing at a large Schmidt number [20], but also in certain single-fractal models of highly turbulent flames [33]. Contrary to the latter models, the front is hypothesized to be another fractal even at smaller length scales $\ell_{i n}<r<\eta_{K}$, rather than a smooth interface. The point is that, under the considered conditions of an infinitely thin and slowly propagating (i.e., $u_{0} \ll u_{K}$ ) front, there is no physical mechanism that can smooth the front surface at scales larger than the Kolmogorov length scale.

Indeed, third, the sole physical mechanism of smoothing small-scale wrinkles on the surface of an infinitely thin front consists of kinematic restoration due to the selfpropagation of the front $[21,22]$. This is the key difference between the present study and a recently developed bifractal model [32] of a highly turbulent reaction wave that has a mixing zone of a finite thickness. For such waves, the inner cut-off scale is controlled by molecular mixing [32]. For an infinitely thin front, the small inner cut-off scale $\ell_{i n}$ is identified as the Gibson scale corresponding to the front velocity $u_{0}$. Therefore, the scale $\ell_{i n}$ is found using the following constraint:

$$
\left|\Delta u\left(\ell_{i n}\right)\right|=u_{0},
$$

where $\Delta u\left(\ell_{i n}\right)$ designates the velocity difference in two points separated by the distance $\ell_{i n}$.

The same constraint is adopted in the classical single-fractal models of turbulent flames [21,22], which address the case of $u_{0}>u_{K}$ and, accordingly, estimate the velocity difference following the Kolmogorov scaling for the inertial interval [4,5], i.e., $\left|\Delta u\left(\ell_{\text {in }}\right)\right| \propto$ $u_{K}\left(r / \eta_{K}\right)^{1 / 3}>u_{K}$. However, under conditions of $u_{0} \ll u_{K}$ examined here, the scale $\ell_{i n}$ belongs to the viscous (dissipation) subrange of the turbulence spectrum. Therefore, the 
difference $\left|\Delta u\left(\ell_{i n}\right)\right|$ should be estimated using the Taylor expansion [4]. Consequently, by retaining the linear term in the expansion, we arrive at

$$
\left|\Delta u\left(\ell_{i n}\right)\right| \approx|\nabla \mathbf{u}| \ell_{i n} \propto \frac{\ell_{i n}}{\sqrt{\bar{\varepsilon} / v}}=\frac{\ell_{i n}}{\tau_{K}} .
$$

Equations (19) and (20) yield

$$
\ell_{\text {in }}=u_{0} \tau_{K}=\frac{u_{0}}{u_{K}} \eta_{K} \ll \eta_{K}
$$

A comparison of Equations (10) and (21) shows that the inner cut-off scale $\ell_{\text {in }}$ is equivalent to the microscale $\ell_{0}$ introduced in Section 2.1. Obviously, the scales $\ell_{i n}$ and $\ell_{0}$ differ from the common Gibson length scale $L_{G}=L\left(u_{0} / u^{\prime}\right)^{3}=\eta_{K}\left(u_{0} / u_{K}\right)^{3}[21,22]$, which characterizes interaction of the front with turbulent eddies from the inertial range.

Fourth, the area of a bifractal surface is evaluated as follows [20,32]:

$$
\begin{aligned}
& A_{f, 1}=A_{0}\left(\frac{L}{\eta_{K}}\right)^{D_{f, 1}-2}, \\
& A_{f}=A_{f, 1}\left(\frac{\eta_{K}}{\ell_{0}}\right)^{D_{f, 2}-2},
\end{aligned}
$$

where subscripts 1 and 2 refer to the large-scale interval of $\eta_{K}<r<L$ and the small-scale interval of $\ell_{0}<r<\eta_{K}$, respectively. In terminology by Sreenivasan et al. [20], $A_{f, 1}$ is the area measured with resolution $\eta_{K}$, and $A_{f}$ is the true front surface area increased jointly by large-scale and small-scale wrinkles.

Substitution of Equation (22) into Equation (23) yields

$$
A_{f}=A_{f, 1}\left(\frac{\eta_{K}}{\ell_{0}}\right)^{D_{f, 2}-2}=A_{0}\left(\frac{L}{\eta_{K}}\right)^{D_{f, 1}-2}\left(\frac{\eta_{K}}{\ell_{0}}\right)^{D_{f, 2}-2}=A_{0} R_{L}^{3\left(D_{f, 1}-2\right) / 4}\left(\frac{u_{K}}{u_{0}}\right)^{D_{f, 2}-2} .
$$

The value of the fractal dimension $D_{f, 2}$ of the small-scale wrinkles can be found by noting that the scales $\ell_{0}<r<\eta_{K}$ are inside the dissipation subrange. Accordingly, the small-scale wrinkles of the front surface fill the space between $\ell_{0}$ and $\eta_{K}$, and, hence, $D_{f, 2}=3$ [34], as proposed by E. Hawkes during discussion with the first author in Dubrovnik in April 2017. For the fractal dimension $D_{f, 1}$ of wrinkles whose scale is larger than $\eta_{K}$, the common value [20-22] of $D_{f, 1}=7 / 3$ may be adopted.

Subsequently, Equations (22) and (24) read

$$
\begin{gathered}
A_{f, 1}=A_{0} \operatorname{Re}_{L}^{1 / 4}, \\
A_{f}=A_{0} R e_{L}^{1 / 4} \frac{u_{K}}{u_{0}}=A_{0} \frac{u^{\prime}}{u_{0}} .
\end{gathered}
$$

Thus, the turbulent consumption velocity is equal to

$$
\bar{u}_{T}=u_{0} \frac{A_{f}}{A_{0}}=u^{\prime} .
$$

Finally, it is worth noting the following point. If we consider the entire small-scale $\left(\ell_{0}<r<\eta_{K}\right)$ fractal to be a broadened front propagating at an increased speed

$$
u_{f, 2}=u_{0}\left(\frac{\eta_{K}}{\ell_{0}}\right)^{D_{f, 2}-2}=u_{0} \frac{\eta_{K}}{\ell_{0}}=u_{K}
$$

then the Gibson length scale for this front is equal to $\eta_{K}$, which, in its turn, is equal to the crossover length scale or the inner cut-off scale for the large-scale $\left(\eta_{K}<r<L\right)$ fractal. This example shows self-consistency of the present estimates of the two inner cut-off scales 
$\ell_{0}$ and $\eta_{K}$, as they both are associated with Gibson scales obtained by comparing the front speed and velocity difference for the appropriate range of the turbulence spectrum. Moreover, the turbulent consumption velocity is again equal to $u^{\prime}$. Indeed,

$$
\bar{u}_{T}=u_{K} \frac{A_{f, 1}}{A_{0}}=u^{\prime}
$$

\section{Discussion}

When small-scale turbulent eddies increase the area of a slowly $\left(u_{0} \ll u_{K}\right)$ propagating front by stretching it, the exponential growth of the area cannot last a long time, because the front packing in a finite volume is limited by annihilation of the front elements in mutual collisions. Accordingly, a stage characterized by a rapidly growing front area and consumption velocity should be followed by another stage, during which the area partly disappears and the consumption velocity decreases. Due to such a physical mechanism, transient effects (oscillations) could appear even during a fully developed stage of the front propagation. Moreover, due to the emphasized physical mechanism and the aforementioned transient effects, the mean turbulent consumption velocity $\bar{u}_{T}$ adjusts itself to the rate of turbulent entrainment, i.e., to the rms turbulent velocity $u^{\prime}$ characterizing large-scale eddies. While the instantaneous front area growth and the increase in a ratio of $u_{T}(t) / u_{0}$ are controlled by the smallest eddies of the Kolmogorov scales, such eddies do not affect the time-averaged front area and turbulent consumption velocity. In some sense, the Kolmogorov eddies behave like the Cheshire cat from Alice in Wonderland.

If the speed $u_{0}$ of a self-propagating infinitely thin front is less than the Kolmogorov velocity $u_{K}$, the front surface should be wrinkled by eddies that are even smaller than the Kolmogorov ones, because the sole mechanism of smoothing the surface wrinkle for the infinitely thin front, i.e., kinematic restoration, can only be efficient at scales smaller than the Kolmogorov length scale $\eta_{K}$ in the considered case. Due to this mechanism, wrinkles with a length scale smaller than $\ell_{0}=u_{0} \tau_{K}=\eta_{K}\left(u_{0} / u_{K}\right) \ll \eta_{K}$ are smoothed out. In other words, the newly introduced length scale $\ell_{0}$ characterizes the smallest possible wrinkles of the surface of a slowly propagating front. Since eddies from both inertial and dissipation ranges of the turbulence spectrum wrinkle the front surface, the surface is expected to be a bifractal with two different fractal dimensions for scales smaller (i.e., $\ell_{0}<r<\eta_{K}$ ) and larger (i.e., $\eta_{K}<r<L$ ) than the crossover scale, which is equal to $\eta_{K}$ under the considered conditions. In spite of the apparent complexity of the above scenario, the mean fluid consumption velocity is simply controlled by the rms turbulent velocity $u^{\prime} \gg u_{0}$ during the late statistically stationary phase of the evolution of the front.

The simple physical scenarios outlined above should be explored in future DNS studies.

Author Contributions: Investigation, V.A.S. and A.N.L.; Methodology, V.A.S. and A.N.L.; Writingoriginal draft, A.N.L.; Writing-review and editing, V.A.S. Both authors have read and agreed to the published version of the manuscript.

Funding: This research was funded by ONERA, Ministry of Education and Science of the Russian Federation (Contract No. 14.G39.31.0001 of 13 February 2017), and the Combustion Engine Research Center (CERC).

Institutional Review Board Statement: Not applicable.

Informed Consent Statement: Not applicable.

Data Availability Statement: All equations are reported in the paper.

Acknowledgments: The first author is grateful to E. Hawkes for fruitful discussion in Dubrovnik in April 2017.

Conflicts of Interest: The authors declare no conflict of interest. 


\section{Nomenclature}

$\begin{array}{ll}A_{0} & \text { initial surface area } \\ A_{F} & \text { area of self-propagating front } \\ A_{M} & \text { material surface area } \\ D & \text { fractal dimension } \\ \bar{d} & \text { mean distance between neighboring front elements } \\ L & \text { integral length scale of turbulence } \\ L_{G} & \text { Gibson scale } \\ \bar{l} & \text { mean thickness of layer consumed by self-propagating front } \\ l_{0}=u_{0} \tau_{K} & \text { newly introduced microscale } \\ \ell_{i n} & \text { inner cut-off scale } \\ \ell_{0 u t} & \text { outer cut-off scale } \\ R e_{L} & \text { turbulent Reynolds number } \\ t & \text { time } \\ \mathbf{u} & \text { velocity vector } \\ u^{\prime} & \text { rms turbulent velocity } \\ u_{0} & \text { speed of a self-propagating front } \\ u_{K} & \text { Kolmogorov velocity } \\ \bar{u}_{T} & \text { mean turbulent consumption velocity } \\ V_{F} & \text { volume occupied by self-propagating front } \\ V_{M} & \text { volume occupied by material surface } \\ \Delta_{F} & \text { streamwise dispersion of self-propagating front } \\ \Delta_{M} & \text { streamwise dispersion of material surface } \\ \varepsilon & \text { viscous dissipation rate of turbulent kinetic energy } \\ \eta_{K} & \text { Kolmogorov length scale } \\ v & \text { kinematic viscosity } \\ \xi & \text { constant } \\ \tau_{K} & \text { Kolmogorov time scale } \\ \tau_{T} & \text { turbulence time scale } \\ & \end{array}$

\section{References}

1. Kuznetsov, V.R.; Sabelnikov, V.A. Turbulence and Combustion; Hemisphere Publ. Corp.: New York, NY, USA, 1990.

2. Chomiak, J. Combustion: A Study in Theory, Fact and Application; Gordon and Breach Science Publishers: New York, NY, USA, 1990.

3. Lipatnikov, A.N. Fundamentals of Premixed Turbulent Combustion; CRC Press: Boca Raton, FL, USA, 2012.

4. Monin, A.S.; Yaglom, A.M. Statistical Fluid Mechanics: Mechanics of Turbulence; The MIT Press: Cambridge, MA, USA, 1975; Volume 2.

5. Frisch, U. Turbulence. The Legacy of A.N. Kolmogorov; Cambridge University Press: Cambridge, UK, 1995.

6. Tsinober, A. An Informal Conceptual Introduction to Turbulence; Springer: Heidelberg, Germany, 2009.

7. Ranzi, E.; Frassoldati, A.; Grana, R.; Cuoci, A.; Faravelli, T.; Kelley, A.P.; Law, C.K. Hierarchical and comparative kinetic modeling of laminar flame speeds of hydrocarbon and oxygenated fuels. Prog. Energy Combust. Sci. 2012, 38, 468-501. [CrossRef]

8. Egolfopoulos, F.N.; Hansen, N.; Ju, Y.; Kohse-Höinghaus, K.; Law, C.K.; Qi, F. Advances and challenges in laminar flame experiments and implications for combustion chemistry. Prog. Energy Combust. Sci. 2014, 43, 36-67. [CrossRef]

9. Bray, K.N.C. Turbulent transport in flames. Proc. R. Soc. Lond. Ser. A Math. Phys. Sci. 1995, 451, 231-256.

10. Lipatnikov, A.N.; Chomiak, J. Effects of premixed flames on turbulence and turbulent scalar transport. Prog. Energy Combust. Sci. 2010, 36, 1-102. [CrossRef]

11. Sabelnikov, V.A.; Lipatnikov, A.N. Recent advances in understanding of thermal expansion effects in premixed turbulent flames. Annu. Rev. Fluid Mech. 2017, 49, 91-117. [CrossRef]

12. Chakraborty, N. Influence of thermal expansion on fluid dynamics of turbulent premixed combustion and its modelling implications. Flow Turbul. Combust. 2021, 106, 753-848. [CrossRef]

13. Lipatnikov, A.N.; Chomiak, J. Molecular transport effects on turbulent flame propagation and structure. Prog. Energy Combust. Sci. 2005, 31, 1-73. [CrossRef]

14. Kerstein, A.R.; Ashurst, W.T. Propagation rate of growing interfaces in stirred fluids. Phys. Rev. Lett. 1992, 68, 934-937. [CrossRef]

15. Chertkov, M.; Yakhot, V. Propagation of a Huygens front through turbulent medium. Phys. Rev. Lett. 1998, 80, 2837-2840. [CrossRef]

16. Mayo, J.R.; Kerstein, A.R. Fronts in randomly advected and heterogeneous media and nonuniversality of Burgers turbulence: Theory and numerics. Phys. Rev. E 2008, 78, 056307. [CrossRef] 
17. Damköhler, G.Z. Der einfuss der turbulenz auf die flammengeschwindigkeit in gasgemischen. Electrochem. Angew. Phys. Chem. 1940, 46, 601-652.

18. Shelkin, K.I. On combustion in a turbulent flow. J. Tech. Phys. 1943, 13, 520-530.

19. Yu, R.; Bai, X.-S.; Lipatnikov, A.N. A direct numerical simulation study of interface propagation in homogeneous turbulence. J. Fluid Mech. 2015, 772, 127-164. [CrossRef]

20. Sreenivasan, K.R.; Ramshankar, R.; Meneveau, C. Mixing, entrainment and fractal dimensions of surfaces in turbulent flows. Proc. R. Soc. Lond. A Math. Phys. Sci. 1989, 421, 79-108.

21. Peters, N. Laminar flamelet concepts in turbulent combustion. Proc. Combust. Inst. 1986, 21, 1231-1249. [CrossRef]

22. Niemeyer, J.C.; Kerstein, A.R. Numerical investigation of scaling properties of turbulent premixed flames. Combust. Sci. Technol. 1997, 128, 343-358. [CrossRef]

23. Kolmogorov, A.N. The local structure of turbulence in incompressible viscous fluid for very large Reynolds number. Dokl. Akad. Nauk SSSR 1941, 30, 299-303.

24. Batchelor, G.K. The effect of homogeneous turbulence on material lines and surfaces. Proc. R. Soc. Lond. A 1952, $213,349-366$.

25. Girimaji, S.S.; Pope, S.B. Propagating surfaces in isotropic turbulence. J. Fluid Mech. 1990, 220, 247-277. [CrossRef]

26. Yeung, P.K.; Girimaji, S.S.; Pope, S.B. Straining and scalar dissipation of material surfaces in turbulence: Implications for flamelets. Combust. Flame 1990, 79, 340-365. [CrossRef]

27. Goto, S.; Kida, S. Reynolds-number dependence of line and surface stretching in turbulence: Folding effects. J. Fluid Mech. 2007, 586, 59-81. [CrossRef]

28. Taylor, G.I. Statistical theory of turbulence. IV. Diffusion in a turbulent air stream. Proc. R. Soc. Lond. A 1935, 151, 421-478. [CrossRef]

29. Lipatnikov, A.N.; Chomiak, J. Turbulent flame speed and thickness: Phenomenology, evaluation, and application in multidimensional simulations. Prog. Energy Combust. Sci. 2002, 28, 1-74. [CrossRef]

30. Townsend, A.A. The Structure of Turbulent Shear Flow, 2nd ed.; Cambridge University Press: Cambridge, UK, 1976.

31. Hawkes, E. Using DNS to understand high Ka premixed combustion and develop predictive combustion models. In Proceedings of the Oral Presentation at Nordita Workshop on Physics of Turbulent Combustion, Stockholm, Sweden, 15 September 2016.

32. Sabelnikov, V.A.; Lipatnikov, A.N. Bifractal nature of turbulent reaction waves at high Damköhler and Karlovitz numbers. Phys. Fluids 2020, 32, 095118. [CrossRef]

33. Thiesset, F.; Maurice, G.; Halter, F.; Mazellier, N.; Chauveau, C.; Gökalp, I. Modelling of the subgrid scale wrinkling factor for large eddy simulation of turbulent premixed combustion. Combust. Theory Model. 2016, 20, 393-409. [CrossRef]

34. Sreenivasan, K.R.; Prasad, R.R. New results on the fractal and multifractal structure of the large Schmidt number passive scalars in fully turbulent flows. Phys. D Nonlinear Phenom. 1989, 38, 322-329. [CrossRef] 